\title{
Dilation Theory and Systems of Simultaneous Equations in the Predual of an Operator Algebra. II
}

\author{
Hari Bercovici ${ }^{1}$, Bernard Chevreau ${ }^{2}$, Ciprian Foias ${ }^{3}$, and Carl Pearcy ${ }^{4}$ \\ ${ }^{1}$ Department of Mathematics, Massachusetts Institute of Technology, Cambridge, \\ Massachusetts 02139, USA \\ ${ }^{2}$ U.E.R. de Mathematique et d'Informatique, Universite de Bordeaux I, France \\ ${ }^{3}$ Department of Mathematics, Indiana University, Bloomington, Indiana 47405, USA \\ 4 Department of Mathematics, The University of Michigan, 347 West Engineering Building, \\ Ann Arbor, Michigan 48109, USA
}

1. This note is a continuation of our earlier paper [3], in which we developed a dilation theory for a certain class of contraction operators acting on a separable, infinite dimensional, complex Hilbert space $\mathscr{H}$. The notation and terminology in what follows is taken from [3]. For the convenience of the reader we recall a few pertinent definitions. The algebra of bounded linear operators on $\mathscr{H}$ is denoted by $\mathscr{L}(\mathscr{H})$. If $T \in \mathscr{L}(\mathscr{H})$, the ultraweakly closed algebra generated by $T$ and $1_{\mathscr{H}}$ is denoted by $\mathscr{A}_{T}$; we recall that $\mathscr{A}_{T}$ can be identified with the dual space of the quotient space $Q_{T}=(\tau c) /{ }^{\perp} \mathscr{A}_{T}$, where $(\tau c)$ denotes the ideal of trace-class operators in $\mathscr{L}(\mathscr{H})$ and ${ }^{\perp} \mathscr{A}_{T}$ is the preannihilator of $\mathscr{A}_{T}$ in $(\tau c)$, under the pairing

$$
\langle A,[L]\rangle=\operatorname{tr}(A L), \quad A \in \mathscr{A}_{T},[L] \in Q_{T} .
$$

The open unit ball in $\mathbb{C}$ is denoted by $\mathbb{D}$, and we write $\mathbb{T}=\partial \mathbb{D}$. The class $\mathrm{A}(\mathscr{H}) \subset \mathscr{L}(\mathscr{H})$ consists of all those absolutely continuous contractions $T$ (i.e., all those contractions $T$ whose unitary part is absolutely continuous or acts on the space (0)) such that the Sz.-Nagy-Foias functional calculus $\Phi_{T}: H^{\infty}(\mathbb{T}) \rightarrow \mathscr{A}_{T}$ is an isometry. If $T \in \mathbb{A}(\mathscr{H})$ then $\Phi_{T}$ is the adjoint of an isometry $\phi_{T}$ of $Q_{T}$ onto the predual $L^{1}(\mathbb{T}) / H_{0}^{1}(\mathbb{T})$ of $H^{\infty}(\mathbb{T})$ (cf. $[3,5]$ ), and via the pair $\left\{\phi_{T}, \Phi_{T}\right\}$, the pair of spaces $\left\{L^{1}(\mathbb{T}) / H_{0}^{1}(\mathbb{T}), H^{\infty}(\mathbb{T})\right\}$ can be identified with the pair $\left\{Q_{T}, \mathscr{A}_{T}\right\}$.

If $x, y \in \mathscr{H}$, we write $x \otimes y$ for the rank-one operator defined, as usual, by $(x \otimes y)(u)=(u, y) x, u \in \mathscr{H}$. Of course, $x \otimes y \in(\tau c)$, and if some $T \in \mathscr{L}(\mathscr{H})$ is given, we write $[x \otimes y]_{Q_{T}}$ (or simply $[x \otimes y]$ when no confusion can result) for the image of $x \otimes y$ in $Q_{T}$. If $n$ is any cardinal number satisfying $1 \leqq n \leqq \aleph_{0}$, we denote by $\mathbb{A}_{n}(\mathscr{H})$ the set of all those $T$ in $\mathbb{A}(\mathscr{H})$ for which every system of simultaneous equations

$$
\left[x_{i} \otimes y_{j}\right]=\left[L_{i j}\right], \quad 0 \leqq i, j<n
$$


(where the $\left[L_{i j}\right]$ are arbitrary elements from $Q_{T}$ ) has a solution $\left\{x_{i}\right\}_{0 \leqq i<n}$, $\left\{y_{j}\right\}_{0 \leqq j<n}$. When no confusion will result, we write simply $\mathbb{A}_{n}$ for $\mathbb{A}_{n}(\mathscr{H})$. In [3] we began the structure theory of the classes $\mathbb{A}_{n}$, and, in particular, the dilation theory of the class $\mathbb{A}_{\mathbb{N}_{0}}$. A primary motivation for the introduction of these classes in [3] was as follows. Let $(B C P)=(B C P)(\mathscr{H})$ denote the set of all those completely nonunitary contractions $T$ in $\mathscr{L}(\mathscr{H})$ for which the intersection $\sigma_{e}(T) \cap \mathbb{D}$ of the essential spectrum of $T$ with $\mathrm{D}$ is sufficiently large that almost every point of $\mathbb{T}$ is a non-tangential limit point of $\sigma_{e}(T) \cap \mathbb{I D}$ (such sets are said to be dominating for $\mathbb{I I}$ ). It was shown in [4] (and also in [7]) that $(B C P) \subset \mathbb{A}_{\aleph_{0}}$, so all of the results obtained in [3] for operators in $\mathbb{A}_{\aleph_{0}}$ apply, in particular, to $(B C P)$-operators. (In fact, in [4], an increasing family $\left\{(B C P)_{\theta}\right\}_{0 \leqq \theta \leqq 1}$ of classes of contractions is introduced, with $(B C P)=(B C P)_{0}$, and it was shown there that $\bigcup_{0 \leqq \theta<1}(B C P)_{\theta} \subset \mathbb{A}_{\aleph_{0}}$.)

In [2] it was shown that all $(B C P)$-operators are reflexive, and the main purpose of this note is to show that all operators in the larger class $\mathbb{A}_{\aleph_{0}}$ are reflexive (Theorem 1.7). This is worthwhile because we show in the third paper [1] of this sequence that many familiar operators belong to $A_{\kappa_{0}}$ and thus are reflexive. In particular, we will show in [1] on the basis of Theorem 1.7 that every weighted unilateral shift $W$ that is a contraction whose spectrum satisfies $\sigma(W) \supset \mathbb{T}$ is reflexive, thus generalizing considerably the results on reflexivity of [8].

We write $\operatorname{Lat}(T)$ for the lattice of invariant subspaces of an operator $T$, and if $\mathscr{M}, \mathscr{N} \in \operatorname{Lat}(T)$ with $\mathscr{M} \supset \mathscr{N}$, so $\mathscr{M} \ominus \mathscr{N}$ is a semi-invariant subspace of $T$, we write $T_{\mathscr{M} \ominus \mathscr{N}}$ for the compression of $T$ to this semi-invariant subspace. We also write $P_{\mathscr{M}}$ for the (orthogonal) projection whose range is a subspace $\mathscr{M}$. Our principal tool is the following result of Robel [7, Proposition 6.1].

Proposition 1.1. Suppose $T \in(B C P)(\mathscr{H}), y \in \mathscr{H}$, and $\varepsilon>0$. Then there exists a subspace $\mathscr{M} \subset \mathscr{H}$ such that $\mathscr{M} \in \operatorname{Lat}(T), T \mid \mathscr{M} \in(B C P)(\mathscr{M}), T_{\mathscr{H} \Theta \mathscr{M}} \in(B C P)(\mathscr{H} \ominus \mathscr{M})$, and $\left\|P_{\mathscr{M}} y\right\|<\varepsilon$.

We will also need the following easy lemma.

Lemma 1.2. Suppose $T$ is a completely nonunitary contraction in $\mathscr{L}(\mathscr{H})$ and $\left\{\lambda_{n}\right\}_{n=1}^{\infty}$ is a sequence in $\mathrm{D}$ that is dominating for $\mathrm{T}$. Suppose also that $\mathscr{M} \in \mathrm{Lat}(T)$ and $T \mid \mathscr{M}$ is a normal diagonal operator with the property that each $\lambda_{n}$ is an eigenvalue of $T \mid \mathscr{M}$ of infinite multiplicity. Then $T \in(B C P)$.

Proof. The hypothesis ensures that each $\lambda_{n}$ belongs to $\sigma_{l e}(T \mid \mathscr{M})$, and since $\sigma_{l e}(T \mid \mathscr{M}) \subset \sigma_{l e}(T)$, we conclude that $\sigma_{e}(T) \cap \mathbb{I D}$ is dominating for $\mathbb{\Pi}$.

The following result is an easy consequence of Proposition 1.1 and Lemma 1.2 .

Proposition 1.3. Suppose $T \in \mathbb{A}_{\aleph_{0}}(\mathscr{H}),\left\{u_{1}, \ldots, u_{n}\right\}$ is any finite subset of $\mathscr{H}$, and $\varepsilon>0$. Then there exists $\mathscr{M} \in \operatorname{Lat}(T)$ such that

(i) both $T \mid \mathscr{M}$ and $T_{\mathscr{H}} \ominus \mathscr{M}$ are $(B C P)$-operators, and

(ii) $\left\|P_{\mathscr{M}} u_{i}\right\|<\varepsilon$ for $i=1, \ldots, n$. 
Proof. Let $\left\{\lambda_{n}\right\}_{n=1}^{\infty} \subset \mathbb{D}$ be dominating for $\mathbb{T}$, and let $N$ be a normal diagonal operator of uniform infinite multiplicity whose eigenvalues constitute the sequence $\left\{\lambda_{n}\right\}_{n=1}^{\infty}$. By Proposition 4.2 of [3] there exist invariant subspaces $\mathscr{M}_{0}$ $\supset \mathscr{K}$ for $T$ such that $T_{\mathscr{M}_{0} \ominus \mathscr{K}}$ is unitarily equivalent to $N$. Thus $N^{*}$ is the restriction to an invariant subspace of $\left(T \mid \mathscr{M}_{0}\right)^{*}$, and it follows from Lemma 1.2 that $\left(T \mid \mathscr{M}_{0}\right)^{*}$ (along with $\left.T \mid \mathscr{M}_{0}\right)$ belongs to $(B C P)\left(\mathscr{M}_{0}\right)$. Let $y_{1}$ be the orthogonal projection of $u_{1}$ onto $\mathscr{M}_{0}$. By Proposition 1.1 there exists $\mathscr{M}_{1} \in \operatorname{Lat}\left(T \mid \mathscr{M}_{0}\right)$ such that $\left(T \mid \mathscr{M}_{0}\right)\left|\mathscr{M}_{1}=T\right| \mathscr{M}_{1}$ is a $(B C P)$-operator and $\left\|P_{\mathscr{M}_{1}} y_{1}\right\|<\varepsilon$. Note that $\mathscr{M}_{1} \in \mathrm{Lat}(T)$ and that $\left\|P_{\mathscr{M}_{1}} u_{1}\right\|=\left\|P_{\mathscr{M}_{1}} y_{1}\right\|$. By an obvious finite induction argument we can find an invariant subspace $\mathscr{M}_{n} \subset \mathscr{M}_{1}$ for $T$ such that $T \mid \mathscr{M}_{n}$ is a $(B C P)$-operator and such that $\left\|P_{\mathscr{M}_{n}} u_{i}\right\|<\varepsilon, i=1, \ldots, n$. Since $T \mid \mathscr{M}_{n} \in \mathbb{A}_{\aleph_{0}}\left(\mathscr{M}_{n}\right)$, we may apply Proposition 4.2 of [3] to $T \mid \mathscr{M}_{n}$ and the operator $N \oplus N$ to conclude the existence of a decomposition

$$
\mathscr{M}_{n}=\mathscr{N}_{1} \oplus \mathscr{N}_{2} \oplus \mathscr{N}_{3} \oplus \mathscr{N}_{4}, \quad \text { where } \mathscr{N}_{1} \text { and } \mathscr{N}_{1} \oplus \mathscr{N}_{2} \oplus \mathscr{N}_{3}
$$

belong to $\operatorname{Lat}\left(T \mid \mathscr{M}_{n}\right)$, and where $\left(T \mid \mathscr{M}_{n}\right)_{\mathscr{N}_{2} \oplus \mathscr{N}_{3}}$ is the operator $N \oplus N$ acting on $\mathscr{N}_{2} \oplus \mathscr{N}_{3}$ in the obvious way. We set $\mathscr{M}=\mathscr{N}_{1} \oplus \mathscr{N}_{2}$. Clearly $\mathscr{M} \in \operatorname{Lat}(T)$, and that $T \mid \mathscr{H} \in(B C P)(\mathscr{H})$ follows as before. Furthermore the restriction of $T_{\mathscr{H}} \ominus \mathscr{A}$ to the invariant subspace $\mathscr{N}_{3}$ is the operator $N$, so, once again by Lemma 1.2 , $T_{\mathscr{H} \ominus \mathscr{M}} \in(B C P)(\mathscr{H} \ominus \mathscr{M})$. Finally, since $\mathscr{M} \subset \mathscr{M}_{n}$, it is obvious that $\left\|P_{\mathscr{M}} u_{i}\right\|<\varepsilon, i$ $=1, \ldots, n$, so the proof is complete.

The next corollary now follows from Proposition 1.3 by the same argument that Robel used to prove [7, Propositions 6.2 and 6.3] from [7, Proposition 6.1].

Corollary 1.4. Suppose $T \in \mathbb{A}_{\aleph_{0}}(\mathscr{H})$. Then $\mathscr{H}$ admits a decomposition $\mathscr{H}=\bigoplus_{n=0}^{\infty} \mathscr{M}_{n}$ such that the operator matrix $\left(T_{i j}\right)$ for $T$ relative to this decomposition is in upper triangular form and satisfies $T_{n n} \in(B C P)\left(\mathscr{M}_{n}\right), 0 \leqq n<\infty$. Furthermore $\mathscr{H}$ admits another decomposition $\mathscr{H}=\bigoplus_{n=-\infty}^{\infty} \mathscr{N}_{n}$ such that the operator matrix $\left(\tilde{T}_{i j}\right)$ for $T$ relative to this decomposition is in upper triangular form and satisfies $\tilde{T}_{n n} \in(B C P)\left(\mathscr{N}_{n}\right),-\infty<n<\infty$.

The following theorem shows that, for operators in $\mathbb{A}_{\aleph_{0}}$, finite systems of simultaneous equations can be solved with reasonable estimates on the distance from the initial data to the solution.

Theorem 1.5. Suppose $T \in \mathbb{A}_{\aleph_{0}}(\mathscr{H}),\left\{\left[L_{i j}\right]\right\}_{1 \leqq i, j \leqq n}$ is a finite set of elements of $Q_{T},\left\{z_{1}, \ldots, z_{m}\right\}$ is an arbitrary finite set of vectors from $\mathscr{H}$, and $\varepsilon>0$. Suppose also that $\left\{x_{1}^{0}, \ldots, x_{n}^{0}\right\}$ and $\left\{y_{1}^{0}, \ldots, y_{n}^{0}\right\}$ are sequences from $\mathscr{H}$ and $\delta>0$ is such that $\left\|\left[L_{i j}\right]-\left[x_{i}^{0} \otimes y_{j}^{0}\right]\right\|<\delta$ for $1 \leqq i, j \leqq n$. Then there exist sequences $\left\{x_{1}, \ldots, x_{n}\right\}$ and $\left\{y_{1}, \ldots, y_{n}\right\}$ of vectors from $\mathscr{H}$ such that

$$
\begin{gathered}
{\left[L_{i j}\right]=\left[x_{i} \otimes y_{j}\right], \quad 1 \leqq i, j \leqq n,} \\
\left\|x_{i}^{0}-x_{i}\right\|<n \delta^{1 / 2}, \quad\left\|y_{i}^{0}-y_{i}\right\|<n \delta^{1 / 2},
\end{gathered}
$$


and

$$
\begin{array}{ll}
\left\|\left[\left(x_{i}^{0}-x_{i}\right) \otimes z_{k}\right]\right\|<\varepsilon, & \left\|\left[z_{k} \otimes\left(x_{i}^{0}-x_{i}\right)\right]\right\|<\varepsilon, \\
\left\|\left[\left(y_{i}^{0}-y_{i}\right) \otimes z_{k}\right]\right\|<\varepsilon, & \left\|\left[z_{k} \otimes\left(y_{i}^{0}-y_{i}\right)\right]\right\|<\varepsilon, \\
& 1 \leqq i \leqq n, 1 \leqq k \leqq m .
\end{array}
$$

Proof. Let $d_{i j}=\left\|\left[L_{i j}\right]-\left[x_{i}^{0} \otimes y_{j}^{0}\right]\right\|, 1 \leqq i, j \leqq n$, and let $\tau$ be a positive number such that

$$
\tau<n\left(\delta^{1 / 2}-\max _{i, j}\left(d_{i j}\right)^{1 / 2}\right)
$$

Let $M>0$ be an upper bound for $\left\|x_{i}^{0}\right\|,\left\|y_{j}^{0}\right\|$, and $\left\|z_{k}\right\|$ for $1 \leqq i, j \leqq n$ and $1 \leqq k \leqq m$. We choose a positive number $\eta$ such that

$$
\eta<\min \left\{\tau / 2, \varepsilon / 3 M, \varepsilon / 3 n \delta^{1 / 2}\right\}
$$

and such that

$$
0 \leqq t, t^{\prime} \quad \text { and } \quad\left|t^{\prime}-t\right|<3 M \eta \quad \text { imply }\left|\sqrt{t^{\prime}}-\sqrt{t}\right|<\tau / 2 n .
$$

(The reason for this choice of $\eta$ will appear later. We choose it now to make it clear that $\eta$ does not depend upon the choice of the upcoming vectors $x_{i}$ and $y_{j}$.) It follows from Proposition 1.3 that there exists $\mathscr{M} \in \operatorname{Lat}(T)$ such that $T \mid \mathscr{M}$ and $S=T_{\mathscr{H}} \Theta \mathscr{M}$ are both $(B C P)$-operators and such that the norm of the (orthogonal) projection onto $\mathscr{M}$ of each of the $2 n+m$ vectors $\left\{x_{1}^{0}, \ldots, x_{n}^{0}\right\}$, $\left\{y_{1}^{0}, \ldots, y_{n}^{0}\right\}$, and $\left\{z_{1}, \ldots, z_{m}\right\}$ is less than $\eta$. We write $x_{i}^{\prime}=P_{\mathscr{H}} \ominus \mathscr{\mu} x_{i}^{0}, 1 \leqq i \leqq n$, and define similarly $y_{j}^{\prime}, 1 \leqq j \leqq n$, and $z_{k}^{\prime}, 1 \leqq k \leqq m$. (The idea of the proof of this theorem should now be clear. We will transfer the equation solving problem to the semi-invariant subspace $\mathscr{H} \ominus \mathscr{M}$, using the fact that $S=T_{\mathscr{H}} \ominus \mathscr{M}$ is a $(B C P)$ operator to solve equations there with "good" bounds, and the smallness of the $\eta$ we have chosen will then give us the estimates we desire.)

For $1 \leqq i, j \leqq n$, let $\left[M_{i j}\right] \in Q_{S}$ be defined by $\left[M_{i j}\right]=\phi_{S}^{-1} \phi_{T}\left(\left[L_{i j}\right]\right)$, and note that the $\left[M_{i j}\right]$ are uniquely determined by the relations

$$
\left\langle S^{p},\left[M_{i j}\right]\right\rangle=\left\langle\lambda^{p}, \phi_{S}\left(\left[M_{i j}\right]\right)\right\rangle=\left\langle T^{p},\left[L_{i j}\right]\right\rangle, \quad p=0,1,2, \ldots
$$

In particular, since the $\left[L_{i j}\right]$ are arbitrary elements of $Q$, for $u, v \in \mathscr{H} \ominus \mathscr{M}$, we have

$$
[u \otimes v]_{Q_{s}}=\phi_{S}^{-1} \phi_{T}\left([u \otimes v]_{Q_{T}}\right)
$$

by virtue of (7), since

$$
\left\langle S^{p},[u \otimes v]_{Q_{S}}\right\rangle=\left(S^{p} u, v\right)=\left(T^{p} u, v\right)=\left\langle T^{p},[u \otimes v]_{Q_{\mathrm{T}}}\right\rangle, \quad 0 \leqq p<\infty .
$$

Let $\alpha=M \eta+\max _{i, j} d_{i j}^{\prime}$, where $d_{i j}^{\prime}=\left\|\left[M_{i j}\right]-\left[x_{i}^{\prime} \otimes y_{j}^{\prime}\right]\right\|_{Q_{s}}$. It now follows from Corollary 6.13 and Remark 6.14 of [3] (applied with $\theta=0$ to the operator $S$ ) that there exist sequences $\left\{x_{1}, \ldots, x_{n}\right\}$ and $\left\{y_{1}, \ldots, y_{n}\right\}$ of vectors in $\mathscr{H} \ominus \mathscr{A}$ such 
that

and

$$
\begin{gathered}
{\left[M_{i j}\right]=\left[x_{i} \otimes y_{j}\right]_{Q_{s}}, \quad 1 \leqq i, j \leqq n,} \\
\left\|x_{i}^{\prime}-x_{i}\right\|<n \alpha^{1 / 2}, \quad\left\|y_{i}^{\prime}-y_{i}\right\|<n \alpha^{1 / 2}, \quad 1 \leqq i \leqq n,
\end{gathered}
$$

$$
\begin{gathered}
\left\|\left[\left(x_{i}^{\prime}-x_{i}\right) \otimes z_{k}^{\prime}\right]\right\|_{Q_{s}}<\varepsilon / 3, \quad\left\|\left[z_{k}^{\prime} \otimes\left(x_{i}^{\prime}-x_{i}\right)\right]\right\|_{Q_{s}}<\varepsilon / 3, \\
\left\|\left[\left(y_{i}^{\prime}-y_{i}\right) \otimes z_{k}^{\prime}\right]\right\|_{Q_{s}}<\varepsilon / 3, \quad\left\|\left[z_{k}^{\prime} \otimes\left(y_{i}^{\prime}-y_{i}\right)\right]\right\|_{Q_{s}}<\varepsilon / 3, \quad 1 \leqq i \leqq n, 1 \leqq k \leqq m .
\end{gathered}
$$

By applying $\phi_{T} \phi_{S}^{-1}$ to (9) and using (8), we see that (1) is satisfied. We will now prove (2) for the $x_{i}^{\prime}$, recalling that $\phi_{S}$ and $\phi_{T}$ are isometries. We have from $(5)$ and $(10)$ that

$$
\left\|x_{i}^{0}-x_{i}\right\| \leqq\left\|x_{i}^{0}-x_{i}^{\prime}\right\|+\left\|x_{i}^{\prime}-x_{i}\right\|<(\tau / 2)+n \alpha^{1 / 2}, \quad 1 \leqq i \leqq n .
$$

Furthermore, from the inequalities

$$
\begin{aligned}
d_{i j}^{\prime} & =\left\|\left[M_{i j}\right]-\left[x_{i}^{\prime} \otimes y_{j}^{\prime}\right]\right\|_{Q_{S}}=\left\|\left[L_{i j}\right]-\left[x_{i}^{\prime} \otimes y_{j}^{\prime}\right]\right\|_{Q_{T}} \\
& \leqq\left\|\left[L_{i j}\right]-\left[x_{i}^{0} \otimes y_{j}^{0}\right]\right\|_{Q_{T}}+\left\|\left[x_{i}^{0} \otimes y_{j}^{0}\right]-\left[x_{i}^{\prime} \otimes y_{j}^{\prime}\right]\right\|_{Q_{T}}
\end{aligned}
$$

we obtain

$$
\begin{aligned}
d_{i j}^{\prime} & \leqq d_{i j}+\left\|\left[x_{i}^{0} \otimes y_{j}^{0}\right]-\left[x_{i}^{\prime} \otimes y_{j}^{\prime}\right]\right\|_{Q_{T}} \\
& \leqq d_{i j}+\left\|\left[x_{i}^{0} \otimes\left(y_{j}^{0}-y_{j}^{\prime}\right)\right]\right\|_{Q_{T}}+\left\|\left[\left(x_{i}^{0}-x_{i}^{\prime}\right) \otimes y_{j}^{\prime}\right]\right\|_{Q_{T}}<d_{i j}+2 M \eta
\end{aligned}
$$

Therefore

$$
\alpha=M \eta+\max _{i, j} d_{i j}^{\prime}<\left(\max _{i, j} d_{i j}\right)+3 M \eta
$$

and from (6) we obtain

$$
\alpha^{1 / 2}<\max _{i, j}\left(d_{i j}^{1 / 2}\right)+\tau / 2 n .
$$

Hence from (12), (13), and (4) we conclude that

$$
\left\|x_{i}^{0}-x_{i}\right\|<\tau / 2+n\left(\max _{i, j} d_{i, j}^{1 / 2}\right)+\tau / 2<n \delta^{1 / 2}, \quad 1 \leqq i \leqq n
$$

as desired. Of course this argument works equally well to prove that $\left\|y_{i}^{0}-y_{i}\right\|<n \delta^{1 / 2}, 1 \leqq i \leqq n$. To conclude the proof of the theorem we content ourselves with proving the first inequality in (3). For $1 \leqq i \leqq n$ and $1 \leqq k \leqq m$ we have

$$
\begin{gathered}
\left\|\left[\left(x_{i}-x_{i}^{0}\right) \otimes z_{k}\right]\right\|_{Q_{T}} \leqq \\
+\left[\left(x_{i}-x_{i}^{\prime}\right) \otimes z_{k}^{\prime}\right]\left\|_{Q_{T}}+\right\|\left[\left(x_{i}-x_{i}^{\prime}\right) \otimes\left(z_{k}-z_{k}^{\prime}\right)\right] \|_{Q_{T}} \\
+\left\|\left[\left(x_{i}^{\prime}-x_{i}^{0}\right) \otimes z_{k}\right]\right\|_{Q_{T}},
\end{gathered}
$$

and using (11), (14), (5) and the fact that $\phi_{S}$ and $\phi_{T}$ are isometries, we obtain

$$
\begin{gathered}
\left\|\left[\left(x_{i}-x_{i}^{\prime}\right) \otimes z_{k}^{\prime}\right]\right\|_{Q_{T}}=\left\|\left[\left(x_{i}-x_{i}^{\prime}\right) \otimes z_{k}^{\prime}\right]\right\|_{Q_{S}}<\varepsilon / 3, \\
\left\|\left[\left(x_{i}-x_{i}^{\prime}\right) \otimes\left(z_{k}-z_{k}^{\prime}\right)\right]\right\|_{Q_{T}} \leqq\left\|x_{i}-x_{i}^{\prime}\right\| \cdot\left\|z_{k}-z_{k}^{\prime}\right\| \leqq n \alpha^{1 / 2} \eta<n \delta^{1 / 2} \eta<\varepsilon / 3,
\end{gathered}
$$


and

$$
\left\|\left[\left(x_{i}^{0}-x_{i}^{\prime}\right) \otimes z_{k}\right]\right\|_{Q_{T}} \leqq\left\|x_{i}^{0}-x_{i}^{\prime}\right\| \cdot\left\|z_{k}\right\| \leqq \eta \cdot M<\varepsilon / 3 .
$$

Thus $\left\|\left[\left(x_{i}^{0}-x_{i}\right) \otimes z_{k}\right]\right\|_{Q_{T}}<\varepsilon$ as desired, and the proof is complete.

The special case of Theorem 1.5 when $n=1$ shows that [2, Proposition 1] is valid for all operators in $\mathbf{A}_{\kappa_{0}}$, and since the proof of $[2$, Corollary 1$]$ only depends on [2, Proposition 1] we have the following.

Corollary 1.6. Suppose $T \in \mathbb{A}_{\aleph_{0}}(\mathscr{H})$, and denote by $\mathscr{W}_{T}$ the smallest subalgebra of $\mathscr{L}(\mathscr{H})$ that contains $T$ and $1_{\mathscr{H}}$ and is closed in the weak operator topology. Then $\mathscr{W}_{T}=\mathscr{A}_{T}$ and the weak operator and ultraweak operator topologies coincide on $\mathscr{A}_{T}$.

It follows from this corollary and a result from [1] that every weighted unilateral shift operator $W$ that is a contraction such that $\sigma(W) \supset \mathbb{\Pi}$ satisfies $\mathscr{W}_{W}=\mathscr{A}_{W}$. This partly answers Question 5 of [8].

Theorem 1.5 also shows that [2, Proposition 2] is valid for all operators in $A_{N_{0}}$, and since the proof of the reflexivity of $(B C P)$-operators used only [2, Proposition 2], we also have the following corollary, which generalizes Theorems 3,4 , and 5 of [2].

Theorem 1.7. Every operator in $\mathbb{A}_{\aleph_{0}}(\mathscr{H})$ is reflexive. In particular, all of the operators in the classes $(B C P)_{\theta}, 0 \leqq \theta<1$, defined in [4] are reflexive.

As mentioned earlier, the utility of Theorem 1.7 will be greatly enhanced by the appearance of [1], because of the large number of operators that turn out to belong to $\mathbb{A}_{\boldsymbol{N}_{0}}$. For the moment we deduce the following corollary of Corollary 1.6 and Theorem 1.7 .

Corollary 1.8. Suppose $T \in C_{00}$ and also $T \in \bigcap_{n=1}^{\infty} \mathbb{A}_{n}(\mathscr{H})$. Then $T$ is reflexive, the algebras $\mathscr{W}_{T}$ and $\mathscr{A}_{T}$ coincide, and the weak operator and ultraweak topologies agree on $\mathscr{A}_{T}$.

Proof. Exner showed in [6] that $\left(\bigcap_{n=1}^{\infty} \mathbb{A}_{n}\right) \cap C_{00} \subset \mathbb{A}_{\mathbf{N}_{0}}$.

This corollary raises the interesting question whether operators in a fixed class $\mathbb{A}_{n}\left(n<\aleph_{0}\right)$ and not in $C_{00}$ have these same properties.

We also note that the upper bounds on $\left\|x_{i}^{0}-x_{i}\right\|$ and $\left\|y_{i}^{0}-y_{i}\right\|$ given by (2) in Theorem 1.5 for all operators in $\mathbb{A}_{\aleph_{0}}$ are better than those given in [4, Corollary 6.11] for $(B C P)_{\theta}$-operators, so Theorem 1.5 generalizes $[4$, Corollary 6.11].

We close this note with a further consequence of Theorem 1.5. If $n \in \mathbb{N}$, we denote by $\tilde{\mathscr{H}}_{n}$ the direct sum of $n$ copies of the Hilbert space $\mathscr{H}$.

Corollary 1.9. Suppose $T \in \mathbb{A}_{\aleph_{0}}, n \in \mathbb{N}$, and $\left\{\left[L_{i j}\right]\right\}_{i, j=1}^{n}$ is a doubly indexed sequence of elements in $Q_{T}$. Then the set of vectors $\left(x_{1}, \ldots, x_{n}\right)$ in $\tilde{\mathscr{H}}_{n}$ for which there exists a vector $\left(y_{1}, \ldots, y_{n}\right)$ in $\tilde{\mathscr{H}}_{n}$ satisfying $(1)$ is dense in $\tilde{\mathscr{H}}_{n}$.

Proof. Let $\tilde{x}_{0}=\left(x_{1}^{0}, \ldots, x_{n}^{0}\right)$ be an arbitrary vector in $\tilde{\mathscr{H}}_{n}$, let $\tau$ be a positive number, and use as initial data in Theorem 1.5 the vectors $\left(\tau x_{1}^{0}, \ldots, \tau x_{n}^{0}\right)$ and 
$(0, \ldots, 0)$ in $\tilde{\mathscr{H}}_{n}$. Then, according to that theorem, there exists a solution $\tilde{x}_{\tau}$ $=\left(x_{1}^{\tau}, \ldots, x_{n}^{\tau}\right), \tilde{y}_{\tau}=\left(y_{1}^{\tau}, \ldots, y_{n}^{\tau}\right)$ of $(1)$ such that

$$
\left\|x_{i}^{\tau}-\tau x_{i}^{0}\right\|<n \delta^{1 / 2}, \quad\left\|y_{i}^{\tau}-0\right\|<n \delta^{1 / 2}, \quad 1 \leqq i \leqq n,
$$

where $\delta$ is any fixed positive number that exceeds $\max \left\|\left[L_{i j}\right]\right\|$. Thus, since for every $\tau>0$, the pair $(1 / \tau) \tilde{x}_{\tau}, \tau \tilde{y}_{\tau}$ is also a solution of (1), and since $\left\|(1 / \tau) \tilde{x}_{\tau}-\tilde{x}_{0}\right\| \rightarrow 0$ by $(15)$, the result follows. In fact, to obtain $\left\|(1 / \tau) \tilde{x}_{\tau}-\tilde{x}_{0}\right\|<\varepsilon$, it suffices to take $\tau=n^{2} \delta^{1 / 2} / \varepsilon^{1 / 2}$, in which case the vector $\tau \tilde{y}_{\tau}$ satisfies $\left\|\tau \tilde{y}_{\tau}\right\|<n^{4} \delta / \varepsilon$.

\section{References}

1. Apostol, C., Bercovici, H., Foias, C., Pearcy, C.: Dilation theory, invariant subspaces, and dual algebras, Preprint.

2. Bercovici, H., Foias, C., Langsam, J., Pearcy, C.: $(B C P)$-operators are reflexive. Michigan Math. J. 29, 371-379 (1982)

3. Bercovici, H., Foias, C., Pearcy, C.: Dilation theory and systems of simultaneous equations in the predual of an operator algebra. I., Michigan Math. J. 30, 335-354 (1983)

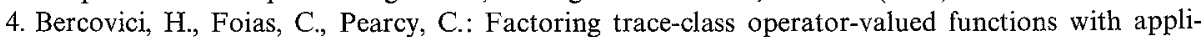
cations to the class $\mathbb{A}_{\mathbb{N}_{0}}$, to appear in J. Operator Theory

5. Brown, S., Chevreau, B., Pearcy, C.: Contractions with rich spectrum have invariant subspaces. J. Operator Theory 1, 123-136 (1979)

6. Exner, G.: Systems of equations in the predual of an operator algebra, the classes $\mathbb{A}_{n}$, and related operators. Thesis, University of Michigan 1983

7. Robel, G.: On the structure of $(B C P)$-operators and related algebras. I. To appear in J. Operator Theory

8. Shields, A.: Weighted shift operators and analytic function theory, Topics in Operator Theory, pp. 49-128. Providence: Amer. Math. Soc. 1974

9. Sz-Nagy, B., Foias, C.: Harmonic analysis of operators on Hilbert space. Budapest: Akademiai Kiado 1970

Received December 21, 1983 